\title{
Millimeter observations and chromospheric dynamics ${ }^{\star}$
}

\author{
M. Loukitcheva ${ }^{1,2}$, S. K. Solanki ${ }^{1}$, M. Carlsson ${ }^{3}$, and R. F. Stein ${ }^{4}$ \\ 1 Max-Planck-Institut für Aeronomie, 37191 Katlenburg-Lindau, Germany \\ 2 Astronomical Institute, St. Petersburg University, 198504 St. Petersburg, Russia \\ 3 Institute of Theoretical Astrophysics, PO Box 1029, Blindern, 0315 Oslo, Norway \\ ${ }^{4}$ Dept. of Physics and Astronomy, Michigan State University, East Lansing, MI 48823, USA
}

Received 5 August 2003 / Accepted 4 February 2004

\begin{abstract}
The intensities of submillimeter and millimeter continua, which are formed in LTE and depend linearly on temperature, may be able to provide a test of models of the Solar chromosphere. We have taken a collection of submillimeter and millimeter wave observed brightness temperatures $T_{\mathrm{b}}$ of the quiet Sun from the literature and compared it with brightness temperatures computed from the standard static models of Fontenla, Avrett and Loeser $(F A L)$ and the dynamic simulations of Carlsson \& Stein $(C S)$. The analysis of the dynamic simulations of Carlsson \& Stein reveals that radio emission at millimeter wavelengths is extremely sensitive to dynamic processes in the chromosphere, if these are spatially and temporally resolved. The most striking result is that the dynamic picture of the solar internetwork chromosphere is consistent with currently available millimeter and submillimeter brightness observations. The spectrum obtained by averaging over the spectra from all time-steps of $C S$ simulations provides a good fit to observed temporally and spatially averaged millimeter data in spite of the absence of a permanent temperature rise at low chromospheric heights in the simulations. This does not by itself rule out the presence of a chromospheric temperature rise as present in the FAL models, since a combination of such models also reproduces the (low resolution) data relatively well. Millimeter observations indicate that using radio techniques it is possible to extend observations of the solar oscillatory component to the heights above those previously observed in the photospheric and low chromospheric spectral lines and submillimeter continuum. For more precise diagnostics of chromospheric dynamics, high temporal and spatial resolution interferometric observations in the millimeter-wavelength region would be particularly useful.
\end{abstract}

Key words. Sun: chromosphere - Sun: oscillations - Sun: radio radiation

\section{Introduction}

After more than 50 years of both theoretical and observational research, the solar chromosphere is still poorly understood. Chromospheric modelling has evolved from the description of the atmosphere in terms of one-dimensional, static, planeparallel, one-component models, to multicomponent models, corresponding to various brightness components from dark cell interior to very bright network (Vernazza et al. 1981; Ayres et al. 1986; Maltby et al. 1986; Fontenla et al. 1993), to multidimensional models of flux tube expansion, treating the atmosphere as consisting of magnetic and non-magnetic parts (cf. Solanki et al. 1991). Most of these models have been static. Observations, however, indicate that the chromosphere is in a dynamic state. The prime example is the quasi-periodic behavior of the $\mathrm{H}$ and $\mathrm{K}$ resonance lines from singly ionized calcium in the internetwork regions. This behavior is referred to as the chromospheric three minute oscillations (Rutten \& Uitenbroek 1991, and references therein). The dynamic behavior of the

\footnotetext{
Send offprint requests to: M. Loukitcheva,

e-mail: marija@peterlink.ru

* Table 1 is only available in electronic form at http://www.edpsciences.org
}

solar chromosphere is also visible in lines and continua observed with the SUMER instrument on-board the SOHO spacecraft (Carlsson et al. 1997; Wilhelm \& Kalkofen 2003). SUMER (Solar Ultraviolet Measurement of Emitted Radiation) observations of the internetwork chromosphere revealed oscillations which appear to be spatially coherent over $3^{\prime \prime}-8^{\prime \prime}$ and occur over about 50 percent of the considered area (Carlsson et al. 1997). The very dynamic behavior manifested by the Ca II resonance lines (Cram \& Damé 1983) even raises the question whether a quasi-static chromosphere exists at all, or if it is fully dynamic by nature. In order to reproduce the observed time-sequence of the intensities of the $\mathrm{Ca}$ II $\mathrm{H}$ and $\mathrm{K}$ lines, a dynamic approach to chromospheric modelling was developed by Carlsson \& Stein $(1992,1995,1997,2002)$.

Standard static atmosphere models (Vernazza et al. 1981; Fontenla et al. 1993) are constructed such that their emergent spectra are in good agreement with the observed temporally and spatially averaged intensities at continuum wavelengths in the EUV between 40 and $140 \mathrm{~nm}$, the Ly $\alpha$ and Ly $\beta$ line intensities and radio brightness temperatures between 0.3 and $20 \mathrm{~mm}$. The intensity in the UV is not a good measure of the local temperature, since the source function is decoupled from the Planck function and in addition the temperature 
a

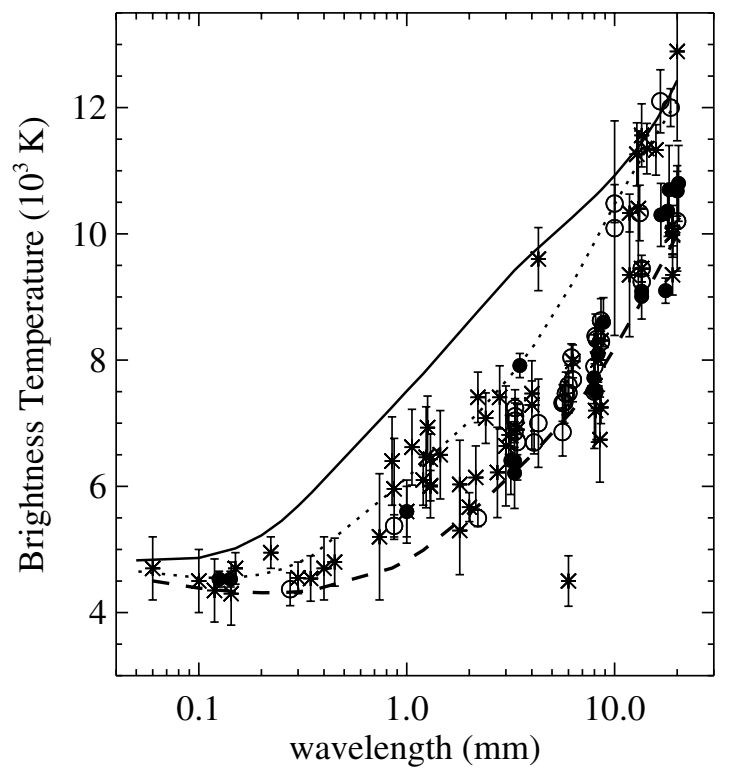

b

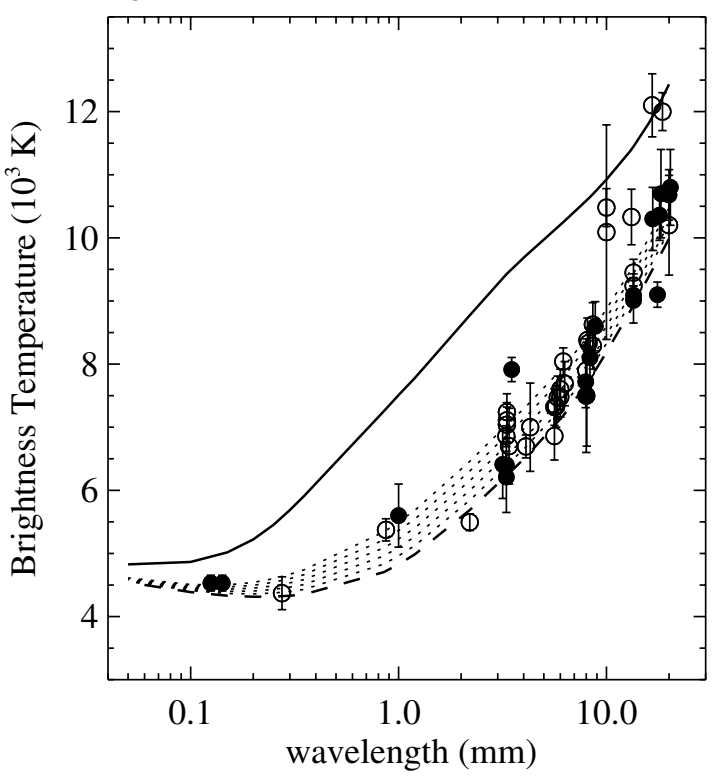

Fig. 1. Observed brightness temperatures, including measurement uncertainties, are plotted vs. wavelength. We distinguish between data obtained around solar cycle minimum (filled circles), solar maximum (open circles) and at intermediate phases (stars). a) $F A L$ models $A, C$ and $F$ are depicted with dashed, dotted and solid curves, respectively. b) Thin dotted curves represent brightness temperature spectra for the $F A L A+F A L F$ combination, with $F A L F$ filling factor values from 0.05 up to 0.25 in steps of 0.05 . Only data obtained around activity maximum and minimum are plotted for clarity.

dependence of the Planck function is closer to exponential than linear. A dynamic model, such as that of Carlsson \& Stein, also reproduces spatially and temporally averaged UV observations, but for a much lower mean kinetic temperature. When averaged over time, the blue or UV continuum intensity has a brightness temperature $T_{\mathrm{b}}$ much higher than the mean kinetic temperature, because the mean intensity preferentially samples the high temperatures (see Carlsson \& Stein 1995). Since UV measurements cannot successfully distinguish between the various modelling approaches, additional diagnostics need to be considered.

The intensities of submillimeter and millimeter continua, which are currently believed to be formed in LTE, depend linearly on temperature and therefore may be able to provide a more sensitive test of models. To ascertain whether this is the case we have taken a collection of submillimeter and millimeter wave observed brightness temperatures, $T_{\mathrm{b}}$, from the literature and compared it with brightness temperatures computed from the standard static models of Fontenla et al. (FAL models) and the dynamic simulations of Carlsson \& Stein (hereafter called the $C S$ model). We consider the question of how well static models and dynamical simulations can reproduce submillimeter and millimeter wavelength observations and discuss the prospects for future observations of chromosphere dynamics at millimeter wavelengths.

\section{Data}

The observed millimeter and submillimeter brightness temperatures, $T_{\mathrm{b}}$, of the quiet Sun at disk center, taken from the original literature are plotted vs. wavelength (including the error bars) in Fig. 1. Open and filled circles refer to solar activity maximum and minimum conditions, respectively; stars represent intermediate phases of the solar cycle (see below). For our study we consider observations compiled from various sources Fürst (1980), Nagnibeda \& Piotrovich (1987), Kislyakov \& Kuznetsova (1977), Pelyushenko (1982) and Fedoseev \& Chernyshev (1998), complemented by the centimeter data of Zirin et al. (1991).

Table 1, which is only available in electronic form contains the observed $T_{\mathrm{b}}$ of the quiet-Sun disk center taken from these sources. Column 1 lists the wavelength in millimeters, Cols. 2 and 3 give the observed brightness temperature and the uncertainties of the observations in Kelvins, respectively. Column 4 lists the date of the observation. Only observations with reported date were analyzed. The phase of the cycle to which a given observation refers is identified in the 5th column of Table 1, with " $\mathrm{M}$ " indicating activity maximum, "m" minimum, and "i" intermediate phases of the cycle. In Col. 6 the beam size $\theta$ of the instrument is given. In the 7 th column we list the method used for the brightness temperature measurement and calibration. The following representation is introduced: "a", "b", "c" indicate comparison of the solar data with the average lunar brightness temperature observed over a lunation, with the brightness of the full Moon and with the brightness of the new Moon, respectively, "d" - eclipse measurements, "e" - use of the method of the "artificial" Moon and black body calibration, "f" - calibration based on vertical atmospheric attenuation, "g" - antenna calibration using a generator, "h" - total flux measurements. The last column lists the references to the original sources.

Note that for most cases, although central disk temperatures are considered, they are averaged over an area of at least 
several square arc minutes, corresponding to the beam size $\theta$ of the instrument. Thus, the brightness temperature spectrum represents the averaged emission of an inhomogeneous solar chromosphere. The observational data used for the current investigation were obtained during solar cycles 19,20 and 21 and, thus, correspond to the chromosphere at different activity phases - from activity minimum to activity maximum (see the dates of the observations listed in Table 1 and the column marked phase). The maximum phase corresponds to an interval of 12 months around the official cycle maximum, the minimum phase to an equal interval centered on activity minimum. The intermediate phase covers everything else. Some of the beam sizes cover a substantial fraction of the solar disc, but nevertheless the reported temperatures qualify for entry in Table 1 because according to the observers the presented values were derived for the quiet Sun disc center $(\mu=1)$ after the contribution due to solar activity was subtracted (for details of the data reduction see the original sources listed in the last column of Table 1). These data do not differ significantly from the rest, so that a test made without them leads to the same general results as discussed in Sect. 4. For the quiet Sun brightness temperatures obtained using methods "a", "b" and "c" we use values re-calibrated according to the accurate reference spectrum of the moon (Pelyushenko 1982).

According to Fig. 1 the observed brightness temperature exhibits a flat minimum at wavelengths shorter than $0.5 \mathrm{~mm}$, which is consistent with the fact that these wavelengths probe the temperature minimum region of a FAL-type model atmosphere. The interval between $1 \mathrm{~mm}$ and $20 \mathrm{~mm}$ displays a smooth increase of $T_{\mathrm{b}}$ from $5 \times 10^{3} \mathrm{~K}$ to $12 \times 10^{3} \mathrm{~K}$ and is the wavelength region which samples much of the chromosphere.

\section{Computation of the brightness temperature}

We compute the brightness temperature $T_{\mathrm{b}}$ at a set of wavelengths covering the same range as the observational data using two sets of model atmospheres. The first set consists of 3 models constructed by Fontenla et al. (1993), hereafter referred to as $F A L$, corresponding to components of the quiet Sun, $A$ (a faint cell center area), $C$ (an average intensity area) and $F$ (a bright area representing the network). At photospheric and lower chromospheric heights the FAL models are almost identical to the models of Vernazza et al. (1981), modified by Avrett (1985) in the $T$ minimum region and by Maltby et al. (1986) in the photosphere. The Skylab EUV data in the 40-140 nm wavelength range allowed the authors to construct 6 sets of atmospheric models corresponding to different brightness components from the low chromosphere to the chromosphere-corona transition region. The temperature structure of the transition region in the $F A L$ models was computed theoretically using the energy-balance equation with hydrogen and helium diffusion included. Fontenla et al. (1993) showed that these models are consistent with observed hydrogen and helium spectra as well.

The other set of models we consider are self-consistent radiation-hydrodynamic simulations of the photosphere and chromosphere (Carlsson \& Stein 1992, 1995, 1997, 2002). The authors studied the dynamics of acoustic waves in the solar

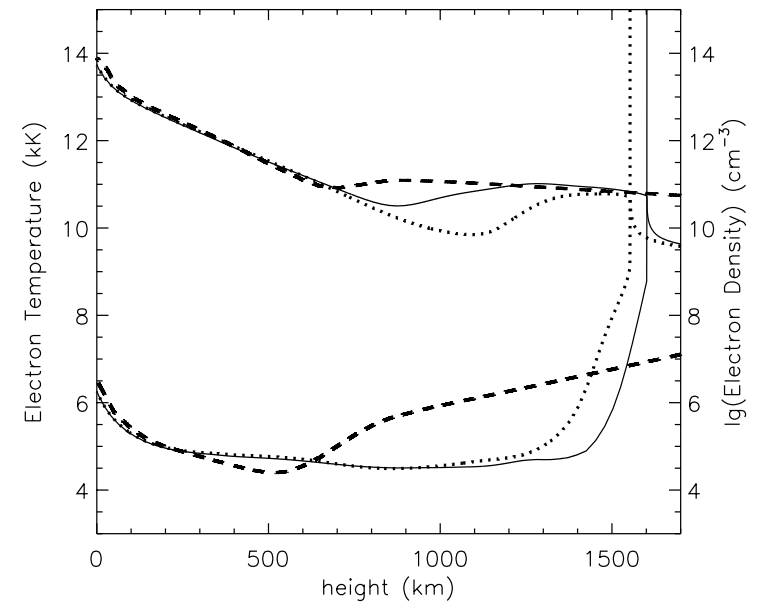

Fig. 2. Temperature and electron density as functions of height for initial (dotted curves) and mean (solid curves) state of the CS atmosphere. The mean is taken for the interval $t=2300-2480 \mathrm{~s}$. For comparison the temperature and electron density of the FALC model are shown with thick dashed curves.

atmosphere using a one-dimensional, non-LTE, radiation hydrodynamic code. The initial atmosphere is in radiative equilibrium above the convection zone without line blanketing and extends $100 \mathrm{~km}$ into the convection zone. As in Carlsson \& Stein (2002), but in contrast with the work reported by Carlsson \& Stein $(1992,1995,1997)$, the initial atmosphere includes a corona and a transition region. This is done through setting the upper boundary at a height of $10 \mathrm{Mm}$ to a temperature of $10^{6} \mathrm{~K}$. Incident radiation from the corona is included which causes ionization in the helium continua in the upper chromosphere and a slow temperature rise above a height of $1 \mathrm{Mm}$ (Fig. 2). Note that in the initial atmosphere the transition region occurs at a lower height than in standard FAL-type models. After the start of the simulations waves are driven through the atmosphere by a subphotospheric piston. The velocity of the piston reproduces an observed $3750 \mathrm{~s}$ sequence of Doppler-shifts of an Fe I line formed at a height of roughly $260 \mathrm{~km}$ above $\tau=1$ at $500 \mathrm{~nm}$, where $\tau$ is the continuum optical depth. Propagating waves increase in amplitude and form shocks above a height of $1 \mathrm{Mm}$. High-amplitude shocks producing temperature differences in excess of $10^{4} \mathrm{~K}$ may originate when a rapidly propagating shock overtakes a preceding, slowly moving shock and they merge. As a consequence the numerical simulations show chromospheric emission enhanced considerably over the radiative equilibrium value. The kinetic temperature significantly exceeds the initial values only episodically during short time intervals, while the mean structure of the dynamic atmosphere (Fig. 2) has a temperature of about $5000 \mathrm{~K}$ throughout most of the chromosphere (up to $1000 \mathrm{~km}$ above $\tau(500 \mathrm{~nm})=1$ ), in contrast to standard empirical models (such as the FAL models), which manifest a temperature increase from a minimum value at about $500 \mathrm{~km}$ above $\tau(500 \mathrm{~nm})=1$.

We have calculated the submillimeter and millimeter radiation emerging from the above static and dynamic models. The radiative transfer was computed under the assumption that radio continuum radiation for wavelengths shorter than 3 centimeter is formed exclusively by free electrons moving in the 
presence of neutral hydrogen atoms and hydrogen ions, i.e. free-free emission (Zlotnik 1968). The equation of radiative transfer is usually written in terms of the intensity $I_{v}$, but at radio frequencies, where $h v \ll k T$, it is convenient to change variables to brightness temperature $T_{\mathrm{b}}$, where

$I_{v}=\frac{2 k T_{\mathrm{b}} v^{2}}{c^{2}}\left[\mathrm{erg} \mathrm{cm} \mathrm{cm}^{-2} \mathrm{~s}^{-1} \mathrm{~Hz}^{-1} \mathrm{sr}^{-1}\right]$

and consider the radiative transfer equation in the form

$\frac{\mathrm{d} T_{\mathrm{b}}}{\mathrm{d} \tau}=T_{\mathrm{e}}-T_{\mathrm{b}}$,

where $T_{\mathrm{e}}=T_{\mathrm{e}}(l)$ is the profile of the kinetic temperature along the light path, $\tau(l)=\int_{l_{0}}^{l} \chi(l) \mathrm{d} l$ is the optical depth, $l$ is geometrical distance along the light path and $\chi$ is the corresponding absorption coefficient, which depends on the effective number of collisions undergone by an electron per unit time (Zheleznyakov 1996), including collisions with protons $(\chi \mathrm{ep})$ and with neutral hydrogen atoms $\left(\chi_{\mathrm{eH}}\right)$. The relative importance of both processes at any wavelength and at any location in the atmosphere depends on the degree of hydrogen ionization. At chromospheric temperatures one cannot neglect encounters between free electrons and neutral hydrogen atoms when the hydrogen ionization fraction is less than $2 \times 10^{-3}$ (Athay 1976). We consider both sources of hydrogen opacity.

The final expression for the vertical optical depth then becomes:

$\mathrm{d} \tau=-\left(\chi_{\mathrm{ep}}+\chi_{\mathrm{eH}}\right) \mathrm{d} h$,

where

$\chi_{\mathrm{ep}} \propto \frac{N_{\mathrm{e}} N_{\mathrm{p}}}{T_{\mathrm{e}}^{3 / 2} v^{2}}$

and

$\chi_{\mathrm{eH}} \propto \frac{N_{\mathrm{e}} N_{\mathrm{H}} T_{\mathrm{e}}^{1 / 2}}{v^{2}}$.

In Eq. (3) $h$ is geometrical height (we consider only the center of the solar disk). In Eqs. (4) and (5) $N_{\mathrm{e}}, N_{\mathrm{p}}$ and $N_{\mathrm{H}}$ are electron, proton and neutral hydrogen number densities, respectively. The expressions for the absorption coefficients are in the forms provided by Zheleznyakov (1996) and Stallcop (1974), which hold also under non-LTE conditions (for Maxwellian electron velocities) because the free-free processes are always fully collisional. Thus, the assumptions for the opacity calculations were Maxwellian distribution of the electron velocity and LTE conditions for the stimulated emission in the absorption coefficient, while the concentrations of electrons, protons and neutral hydrogen atoms were taken from Fontenla et al. (1993) for FAL models and were derived using the non-LTE radiative transfer code MULTI (Carlsson 1992) for the CS model.

The solution of Eq. (2) on a discrete grid can be expressed in terms of $T_{\mathrm{e}}$ as (Hagen 1951):

$T_{\mathrm{b}}^{v}=\sum_{r=1}^{n}\left(1-\exp ^{-\chi_{\mathrm{r}} \Delta h}\right) T_{\mathrm{e}}^{r} \exp ^{-\sum_{s=1}^{r-1} \chi_{\mathrm{s}} \Delta h}$, where $r=1$ corresponds to the layer at the top of the atmosphere, $\Delta h$ is grid distance. The items within the sum represent the contribution of various layers to the emergent intensity of the radiation. We will refer to them as the values of the contribution function (CF) to $T_{\mathrm{b}}$ (not normalized). To maintain the stability of the numerical solution (Eq. (6)) in the presence of shocks we have interpolated the $C S$ model atmospheres onto a finer equidistant grid. Tests revealed that a completely stable solution was reached for interpolated atmospheres with 30000 depth points. The results of the transfer calculations at millimeter wavelengths were checked using the non-LTE radiative transfer code MULTI (Carlsson 1992) and were found to be congruent to high accuracy.

\section{Results and discussion}

\subsection{FAL models}

The millimeter and submillimeter radiation was calculated for the quiet Sun $F A L$ models $A, C$ and $F$ using Eqs. (3)-(6) for the tabulated values of $T_{\mathrm{e}}(h), N_{\mathrm{e}}(h), N_{\mathrm{p}}(h)$ and $N_{\mathrm{H}}(h)$ from Fontenla et al. (1993) and is depicted in Fig. 1 together with the observational data. Clearly, the relatively small number of observations at a given wavelength, the scatter of the data points and the size of the error bars preclude searching for an appropriate model. We nonetheless expect that the model $F A L C$, usually referred to as an average quiet Sun model, provides a good fit to the spatially and time averaged data. However, at wavelengths longer than $1 \mathrm{~mm}$ the FALC model manifests brightness temperatures higher than the observed values. The majority of the observed $T_{\mathrm{b}}$ values lies between the curves due to the $F A L C$ and FALA model, the latter corresponding to the internetwork. The $F A L F$ model produces brightness temperatures much higher than observed at all wavelengths above $0.1 \mathrm{~mm}$. Consequently the millimeter-wave data are not compatible with UV spectra within the context of single-component FAL models: the UV data require a warmer model than the microwave data. A combination of a cell interior $(F A L A)$ and a network model $(F A L F)$ looks more promising, as can be judged from Fig. $1 \mathrm{~b}$. The dotted curves have $T_{\mathrm{b}}=\alpha T_{\mathrm{b}}^{F A L F}+(1-\alpha) T_{\mathrm{b}}^{F A L A}$ with $\alpha$, the filling factor of $F A L F$, increasing from 0.05 to 0.25 in steps of 0.05 . Although by eye all $\alpha$ between 0 and 0.25 provide fits of approximately equal quality, the $\chi^{2}$ is smallest for $\alpha=0.18$ and reaches the value of 0.5 . For comparison, FALA, FALC and FAL F separately produce $\chi^{2}=1.59$, $\chi^{2}=5.54$ and $\chi^{2}=23.23$, respectively. Further details on the formation of millimeter radiation in such models is given in Sect. 4.2, in connection with the Carlsson \& Stein models.

\subsection{CS model}

We have analyzed a time series of atmospheres produced by the dynamic simulations of Carlsson \& Stein. The time series covers 18 wave periods of approximately $180 \mathrm{~s}$ each, with a cadence of $10 \mathrm{~s}$ between the snapshots. The time series we analyzed starts at $t=400 \mathrm{~s}$ after the beginning of the simulations and ends at $t=3600 \mathrm{~s}$. Two wave periods with $2 \mathrm{~s}$ cadence which cover the time-spans $t=500-680 \mathrm{~s}$ 

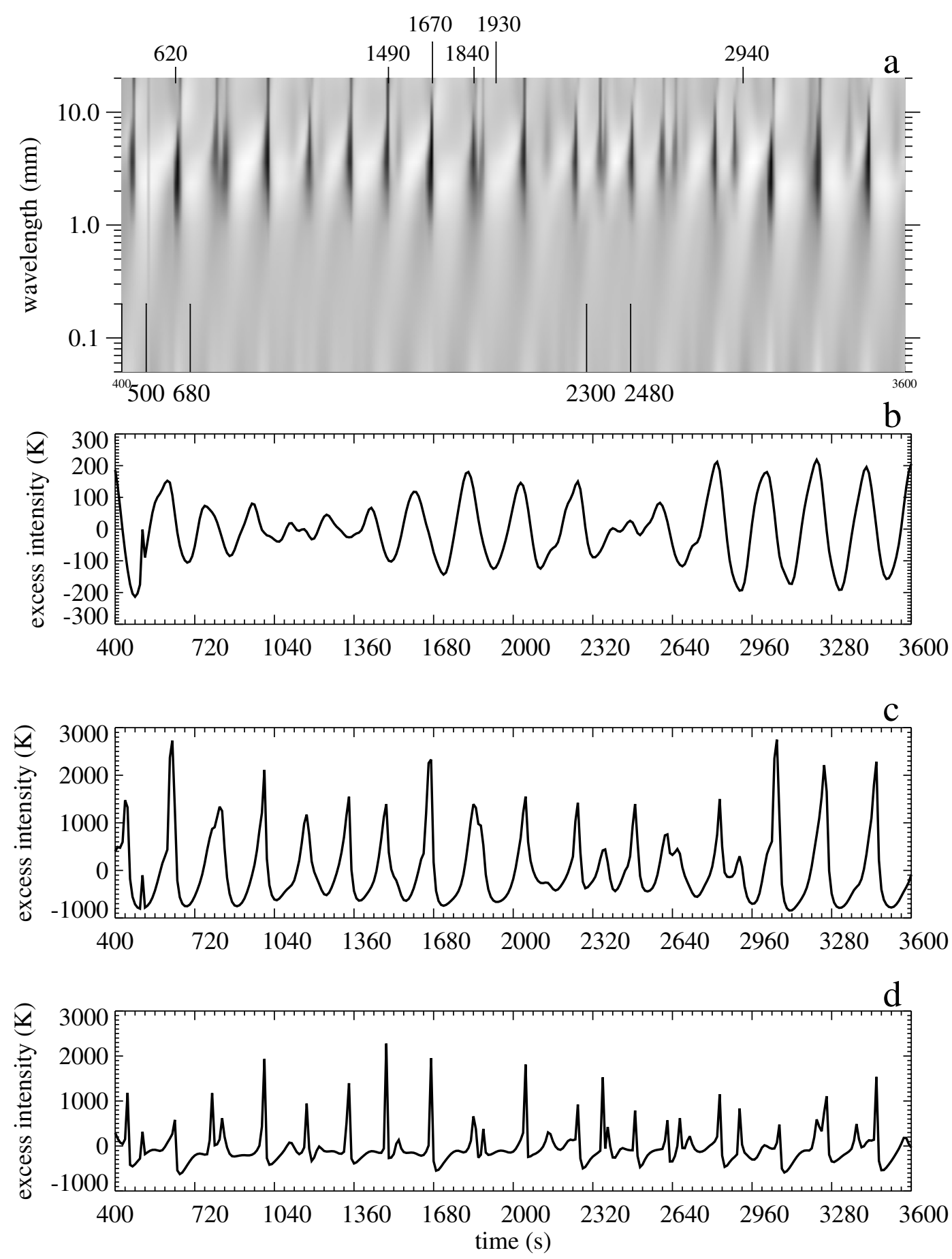

Fig. 3. The evolution of the millimeter spectrum with time (counted from the beginning of the simulation). Plotted is the excess spectrum, i.e. the instantaneous spectrum from which the time-averaged spectrum has been subtracted. The marked times are discussed in the text. a) Negative gray-scale representing excess intensity as a function of time and wavelength. b-d) Excess intensity at the wavelengths $0.05 \mathrm{~mm}$, $1 \mathrm{~mm}$ and $10 \mathrm{~mm}$, respectively, vs. time.

and $t=2300-2480 \mathrm{~s}$ were examined in greater detail. Figure 3 shows the excess intensity (brightness temperature) as a function of wavelength and time. The excess brightness temperature is the brightness temperature of a snapshot minus the time averaged brightness temperature. In Fig. 3a black represents the brightest events. Light curves at 3 wavelengths are plotted in Figs. 3b-d. Wave periods of approximately $180 \mathrm{~s}$ can be clearly distinguished. Equally striking is the difference from one wave period to another. Wave periods which show extremely high excess intensity, like the period $t=500-680 \mathrm{~s}$ chosen for detailed analysis and marked in Fig. 3, are usually characterized by the presence of merging shocks. There are also wave periods which exhibit little excess intensity (see the interval $t=2300-2480 \mathrm{~s}$ in Fig. 3). From the figure it is clear that the peaks in brightness become narrower towards longer wavelengths and also higher, before their amplitude decreases again at the largest 
a

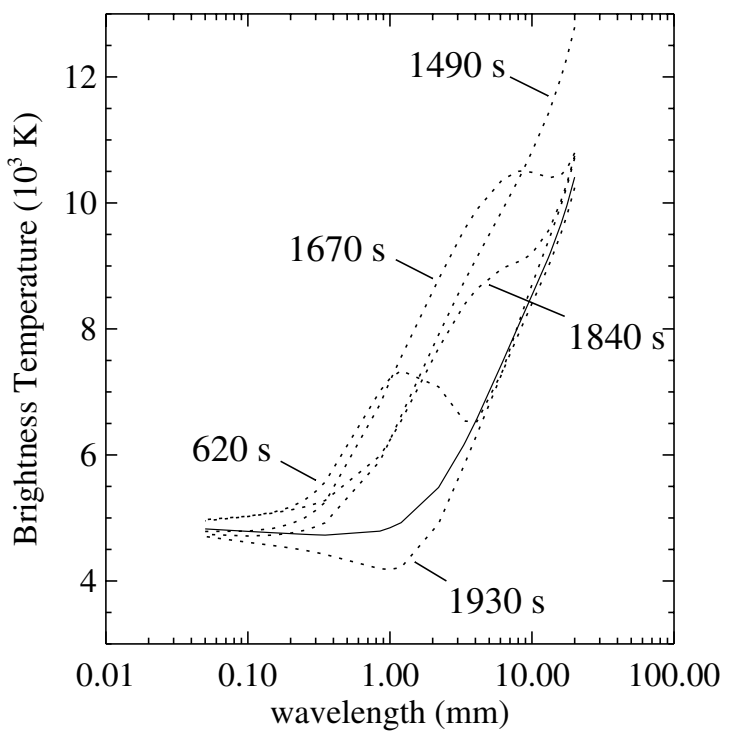

$\mathrm{b}$

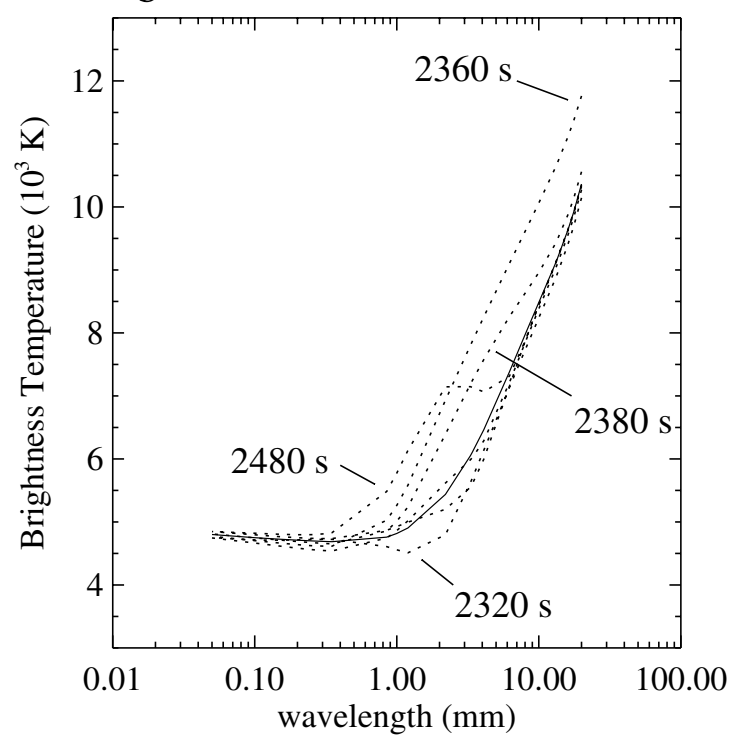

Fig. 4. Model millimeter and submillimeter spectra. a) Dotted curves: brightness temperature spectra of extreme snapshots selected from the time series ( $t=400-3600 \mathrm{~s})$ of the $C S$ simulation. The solid curve corresponds to the spectrum averaged over the whole time series. b) Spectra sampling the period $t=2300-2480 \mathrm{~s}$ (dotted curves) and averaged over this wave period (thick solid curve).

considered wavelengths. The greatest excess brightness is achieved between 1 and $8 \mathrm{~mm}$. At the longest wavelengths most fluctuations in brightness are small, with only occasional short and intense brightening. This can also be seen from Fig. 4a, in which brightness temperature spectra of several extreme states of the atmosphere, marked in Fig. 3, are plotted, together with the spectrum averaged over the whole analyzed time series. It is remarkable that over much of the considered wavelength range the average spectrum lies rather close to the spectrum at quiescent phases. The reason for this can be deduced from Fig. 3. The brightening episodes are sufficiently short that the quiescent spectrum dominates. For comparison we have plotted the spectrum at different times within a single wave period $t=2300-2480 \mathrm{~s}$ in Fig. 4b. Since the shocks in this interval were not particularly strong, the brightening is considerably less marked than for some of the snapshots selected for Fig. 4a.

In order to obtain a better understanding of how the radiation samples the dynamic atmosphere in the presence of shocks we consider 3 snapshots in greater detail before discussing the time average. In Fig. 5 we plot the $T_{\mathrm{e}}$ and $N_{\mathrm{e}}$ stratifications for these 3 times (we have chosen $580 \mathrm{~s}, 620 \mathrm{~s}$ and $1670 \mathrm{~s}$ ) as well as contribution functions for 4 sample wavelengths. The chosen snapshots show the atmosphere in extreme states. At $580 \mathrm{~s}$ the atmosphere is close to the quiescent phase. From Figs. 5a and $7 \mathrm{a}$ it is seen that the atmosphere is in a state somewhat similar to the FAL-type model $A$. However, the presence of a local depression in the temperature profile narrows the range of formation heights and moves it to higher values at middle millimeter wavelengths. At $620 \mathrm{~s}$ there is a shock moving through the atmosphere, which produces localized heating and a density enhancement. The transition region is located at around $1500 \mathrm{~km}$. The atmosphere at $1670 \mathrm{~s}$ is in an extreme state where the height of the shock and the height of the transition region are close to each other. This situation can be described as a low-lying transition region at around $1300 \mathrm{~km}$. The effective emitting region is very sharply defined and is placed at nearly the same heights for all wavelengths in the millimeter range.

For the purposes of discussion, we separate the submillimeter-millimeter wavelength range into 3 sub-ranges and consider them individually.

$\lambda<0.5 \mathrm{~mm}$ : the height of formation (center-of-gravity of the contribution function) is located at $400-600 \mathrm{~km}$ above the $\tau(500 \mathrm{~nm})=1$ level in a $F A L$-type model (Figs. 5b, d and f). In the numerical simulations the temperature and brightness temperature is very moderately time-dependent (Figs. 3 and 5). Although the contribution function is affected somewhat by the evolving structure of the atmosphere (Figs. 5b, d, f and 7b), it remains in the same range of heights as for the FAL-type models. Compared to other wavelengths these changes are small.

$0.5 \leq \lambda<8 \mathrm{~mm}$ : radio emission in this range of wavelengths is most sensitive to the propagation of acoustic waves through the atmosphere. The brightness temperature contribution functions are extremely time-dependent, following changes in the atmospheric parameters $T_{\mathrm{e}}$ and $N_{\mathrm{e}}$. The emission is generated over a range of heights close to where shocks are formed and for some snapshots exhibits a doublypeaked contribution function (see Figs. $5 \mathrm{~b}, \mathrm{~d}$ and $\mathrm{f}$ for $\lambda=$ $3.4 \mathrm{~mm}$ ), with one peak around the height of the effective emitting region and another at a shock at smaller optical depth.

$\lambda \geq 8 \mathrm{~mm}$ : radio emission at these wavelengths is generated in the transition region, which is characterized in these 1-D models by a rapid increase in temperature. The effective emitting region is sharply defined due to the fact that the absorption coefficient varies rapidly with height (Figs. 5b, d and $\mathrm{f}$ ). The height at which the radiation is produced changes considerably over a wave period, but since the $T_{\mathrm{e}}$ and $N_{\mathrm{e}}$ at the transition region remain otherwise unchanged most of the 

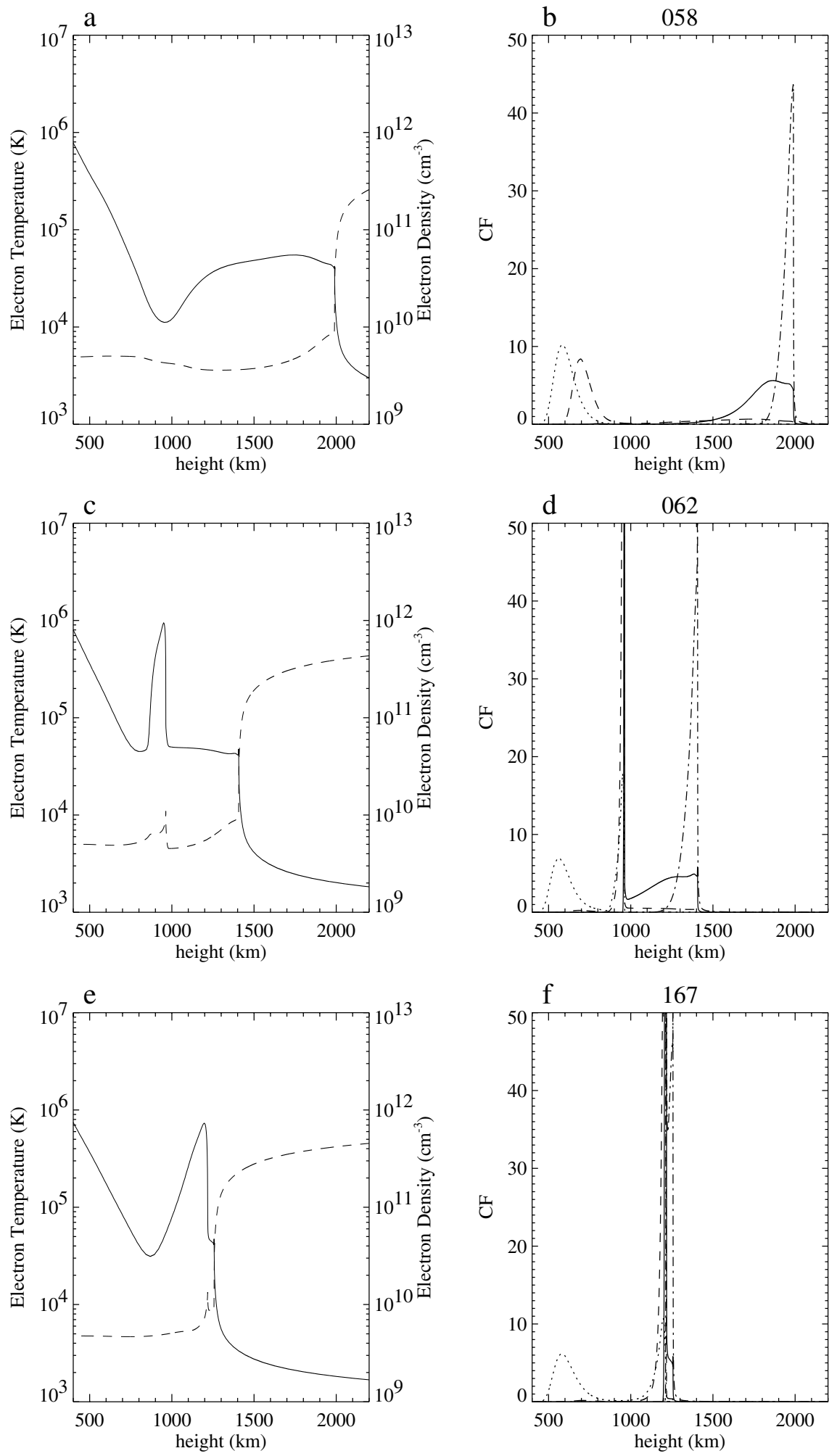

Fig. 5. Model atmospheres and contribution functions for $C S$ numerical simulations referring to $t=580 \mathrm{~s}, t=620 \mathrm{~s}$ and $t=1670 \mathrm{~s}$ from the start of the simulation. a), c), e) Electron temperature (left axis, dashed curve) and electron number density (right axis, solid curve) are presented for the selected snapshots. b), d), f) The corresponding brightness temperature contribution functions for wavelengths $0.35 \mathrm{~mm}$ (dotted curve), $1 \mathrm{~mm}$ (dashed curve), $3.4 \mathrm{~mm}$ (solid curve) and $10 \mathrm{~mm}$ (dot-dashed curve) are shown.

time, the brightness temperature at the longest wavelengths exhibits little time dependence (see Fig. 3). Since in the model a corona with a temperature of $10^{6} \mathrm{~K}$ placed at $10^{6} \mathrm{Mm}$ is treated as the upper boundary, the transition region-like feature is probably not sufficiently realistic and the result for long millimeter wavelengths needs to be considered with caution.

Because of the large radio beamwidth of currently available data it is impossible to resolve the individual oscillating 

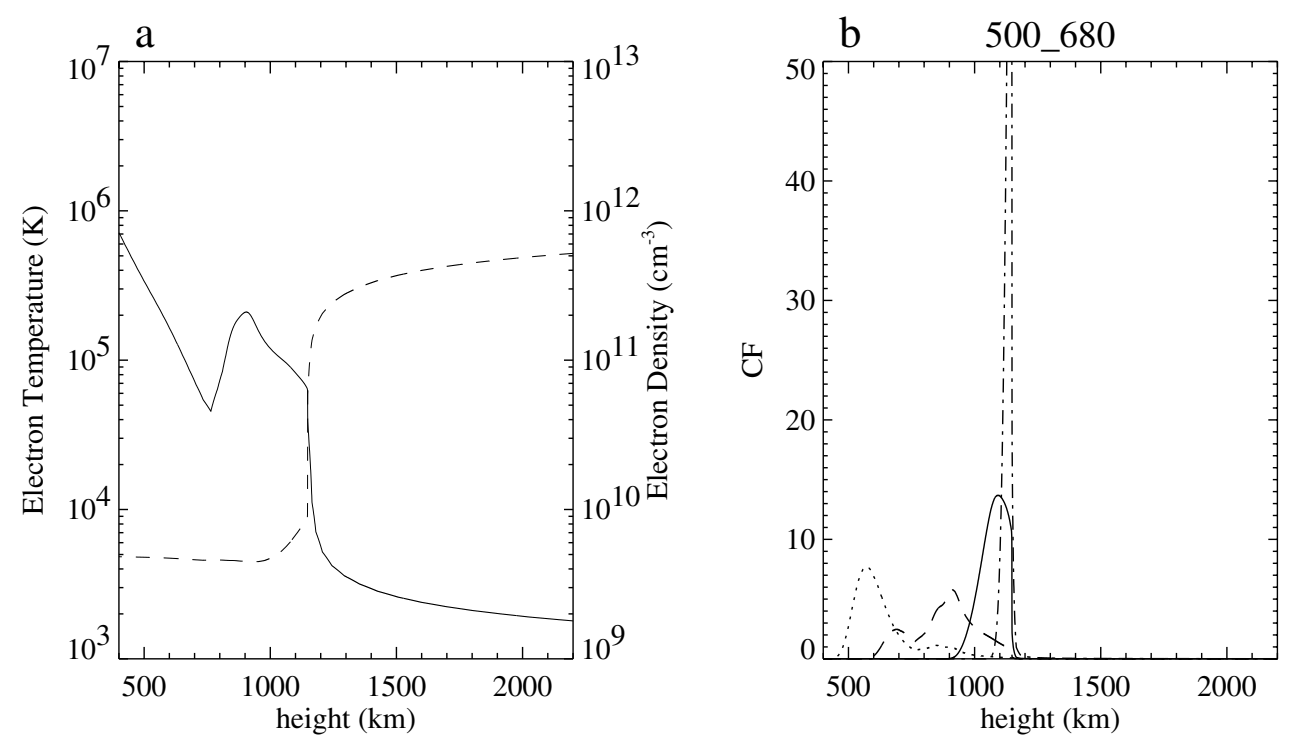

Fig. 6. a) Electron temperature (left axis, dashed curve) and electron number density (right axis, solid curve) are presented for the mean $C S$ model derived for the time-span $t=500-680 \mathrm{~s}$. The corresponding brightness temperature contribution functions for wavelengths $0.35 \mathrm{~mm}$ (dotted curve), $1 \mathrm{~mm}$ (dashed curve), $3.4 \mathrm{~mm}$ (solid curve), $10 \mathrm{~mm}$ (dot-dashed curve) are shown in b).

elements, which typically lie below supergranular scales (cf. Rutten \& Uitenbroek 1991). For a typical beam width $\theta \sim 2^{\prime}$ and the temporal averaging inherent in the observations considered we do not expect the oscillations to produce significant scatter in the observations (differences in beam-size and methods may have a larger influence). Therefore, for the comparison of the dynamic simulations with observational data we used the brightness temperature spectra derived by averaging over spectra from all the time steps of the complete analyzed time series $t=400-3600 \mathrm{~s}$. The resulting $T_{\mathrm{b}}$ as a function of $\lambda$ is plotted in Fig. 8 (solid curve), which is identical to the thick solid curve in Fig. 4a. We have also analyzed the averaged spectra for the wave periods $t=2300-2480 \mathrm{~s}$ and $t=500-680 \mathrm{~s}$, at a cadence of $0.1 \mathrm{~s}$. The brightness temperature spectra derived by averaging over different time periods show an almost identical dependence of $T_{\mathrm{b}}$ on $\lambda$ as the averaged spectrum, and have not been plotted in Fig. 8 since they would be almost indistinguishable from the solid curve. The great similarity between the 3 spectra suggests: a) averaged over a full wave period the millimeter wave spectrum does not depend on the strength of the shock; b) a cadence of $10 \mathrm{~s}$ is sufficient for reliable averaging.

For the two latter periods we also derived mean models by averaging the atmospheres over a full wave period of $180 \mathrm{~s}$ with a cadence of around $0.1 \mathrm{~s}$. The mean was taken of the thermodynamic variables at a constant column mass, which corresponds to a Lagrangian mean. The resulting $T_{\mathrm{b}}$ of the mean models can be seen in Fig. 8 represented by thin dashed $(t=2300-2480 \mathrm{~s})$ and dotted $(t=500-680 \mathrm{~s})$ curves. The spectra of the mean models of the two wave periods are surprisingly similar to each other and to the averaged spectra, even at longer wavelengths. There the wave period with the stronger shock $(t=500-680 \mathrm{~s})$ produces slightly larger brightening. At shorter millimeter wavelengths (between 0.3 and $5 \mathrm{~mm}$ ) the averaged spectrum is brighter than the spectrum computed from the mean atmosphere, because in an averaged model this radiation comes from the lower chromosphere where the mean temperature shows no rise (see Fig. 6). However, as Fig. 5 shows, the radiation at 3.4 and $10 \mathrm{~mm}$ obtains its major contribution from the height at which $T_{\mathrm{e}}$ does exhibit higher values. This is associated with the enhanced $N_{\mathrm{e}}$ at these heights and the $N_{\mathrm{e}}$ dependence of the optical depth. Figure 8 would seem to suggest that $T_{\mathrm{b}}$ depends linearly on a linear combination of $T_{\mathrm{e}}$ and $N_{\mathrm{e}}$, although from Sect. 3 and Figs. 4-6 this is clearly not the case. This seeming linearity is produced by a complex interplay between the actual dependence on $T_{\mathrm{e}}$ and $N_{\mathrm{e}}$ and changing heights of formation as the structure of the atmosphere varies.

Note that every snapshot during the periods being considered exhibits an increase of $T_{\mathrm{b}}$ with $\lambda$ for $\lambda \geq 1 \mathrm{~mm}$. The mean model obtained from the dynamic simulations, i.e. the average of the $T_{\mathrm{e}}$ and $N_{\mathrm{e}}$ stratifications over a wave period, which has a constant temperature throughout most of the chromosphere and a temperature rise only in the upper chromosphere (Fig. 2), also produces brightness temperatures increasing with wavelength. This is surprising, since our calculations are in LTE and at radio wavelengths the source function is linear in temperature. A careful look at the contribution functions for different wavelengths reveals that between roughly 1 and $5 \mathrm{~mm}$ they have two peaks for many of the snapshots, as well as for the mean model. For instance, in Figs. 5 and $6 \mathrm{~b}$ one of the peaks lies at the shock, the other in the heated, "chromospheric" portion. As the wavelength increases, the strength of the higher peak, sampling hotter gas, increases, leading to a higher $T_{\mathrm{b}}$.

One reason why the microwave radiation does not follow the strongly fluctuating temperature at a given height, but rather is formed in the region following the shock through the atmosphere, is that the shocks are associated with a significantly higher $N_{\mathrm{e}}$, which leads to an increased optical depth (Eqs. (4) and (5)), so that $\tau=1$ often lies in the vicinity of the shock (e.g., Fig. 5). In Fig. 9 we compare the averaged spectrum resulting from the $C S$ simulation (dashed curve) with the data. 

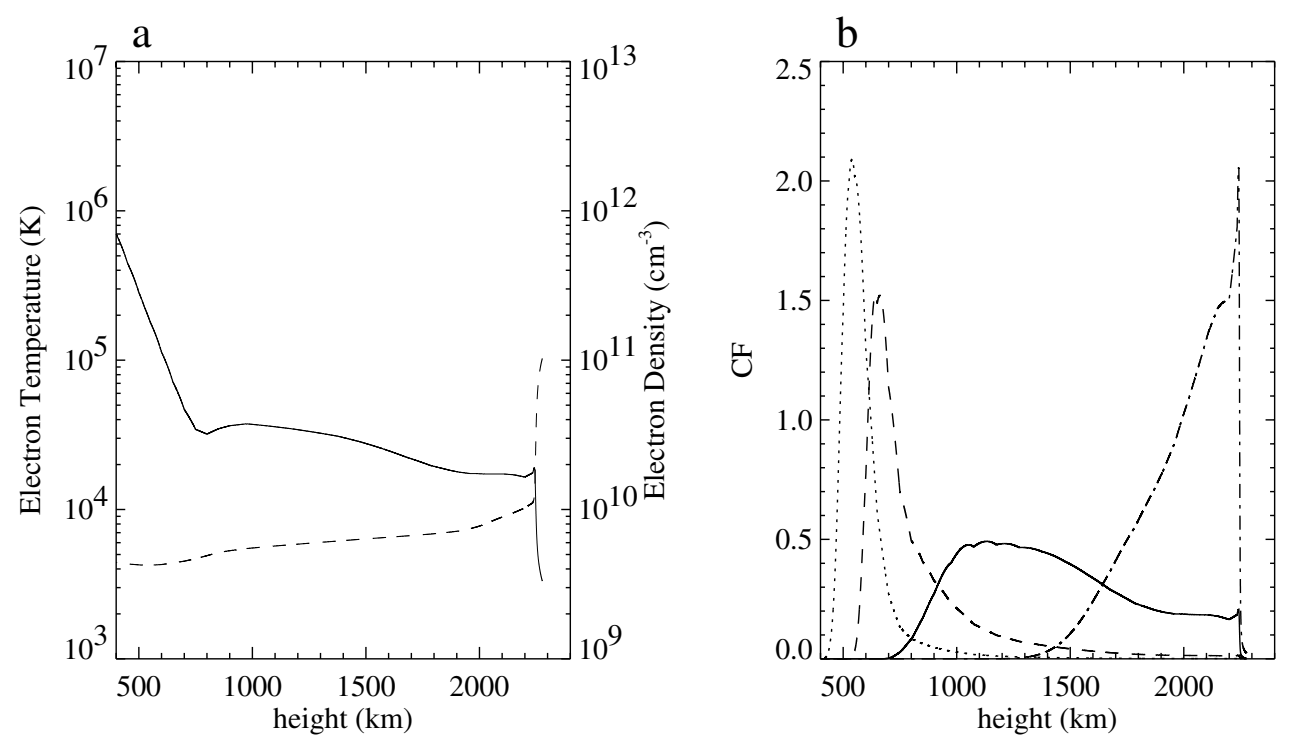

Fig. 7. a) Electron temperature (left axis, dashed curve) and electron number density (right axis, solid curve) are presented for the model $F A L A$. The corresponding brightness temperature contribution functions for wavelengths $0.35 \mathrm{~mm}$ (dotted curve), $1 \mathrm{~mm}$ (dashed curve), $3.4 \mathrm{~mm}$ (solid curve), $10 \mathrm{~mm}$ (dot-dashed curve) are shown in $\mathbf{b}$ ).

Although a strong increase in brightness temperatures occurs at a time-scale of several snapshots (see $T_{\mathrm{b}}(\lambda)$ for $t=2360 \mathrm{~s}$, $t=2480 \mathrm{~s}$ in Fig. 4), the averaged $T_{\mathrm{b}}$ spectrum as well as the $T_{\mathrm{b}}$ spectrum computed from the averaged model provide a good fit to the observational data, of a quality similar to that provided by FALA.

The quiet Sun is composed of at least two components, a bright network associated with the magnetic network located at the edges of supergranular cells, and a darker internetwork associated with cell interiors. The $C S$ model and FALA represent the cell interiors. The observations on the whole have poor spatial resolution and are averaged over both components. Therefore we calculated $T_{\mathrm{b}}$ spectra for various values of the filling factor of $F A L$ model $F$, which is taken to represent the network. The resulting brightness temperature of the form: $T_{\mathrm{b}}=\alpha T_{\mathrm{b}}^{F A L F}+(1-\alpha) T_{\mathrm{b}}^{F A L A}$ or $C S$, with $\alpha$ being the filling factor in the range $\alpha=0.05$ to 0.25 is plotted in Fig. 1 for model FALA (thin dotted curves) and in Fig. 9 for the average over the complete analyzed interval of spectra from the CS model (thin dotted curves). In order to determine which filling factor and which model combination is the most appropriate we calculated from the $\chi^{2}$ deviation of averaged model spectra corresponding to different $\alpha$ the observations obtained with instruments which have $\theta \leq 15^{\prime}$. We found that for the model $C S$ the observational data are best reproduced for a filling factor of 0.12 when a reduced $\chi^{2}$ value of 1.0 is reached, while the model FALA provides the best fit for a filling factor of 0.18 at a reduced $\chi^{2}$ value of 0.5 . The $C S$ model by itself produces $\chi^{2}=1.41$.

\section{Conclusions}

In this investigation we have used millimeter and submillimeter observations of the quiet Sun brightness temperature as a test of two rather different views of the structure and dynamic state of the solar chromosphere. The first of them, represented

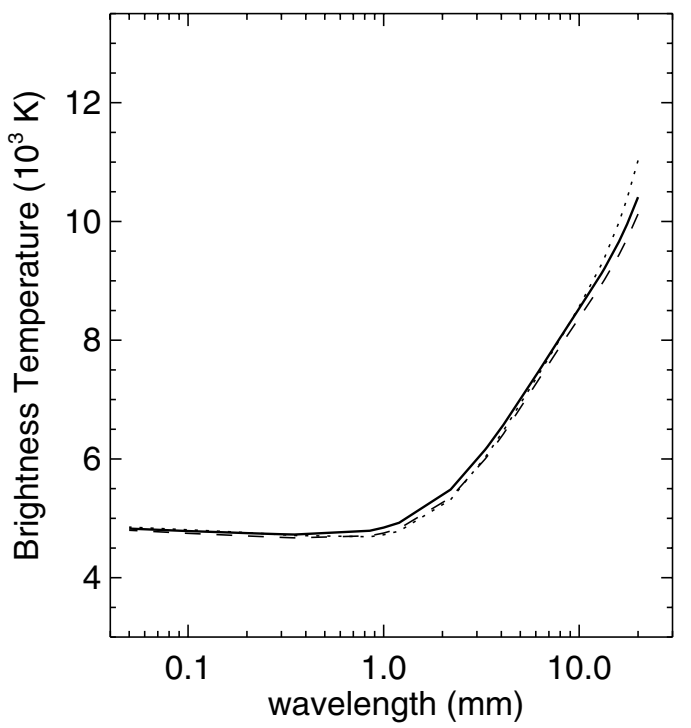

Fig. 8. Brightness temperature spectra averaged over the whole simulated time series, $t=400-3600 \mathrm{~s}$ (thick solid curve). For comparison, the brightness temperature spectra calculated for models averaged over the intervals $t=2300-2480$ and $t=500-680 \mathrm{~s}$, derived using all time steps of the simulation are also plotted (thin dashed and dotted curves).

by Carlsson \& Stein, considers the chromosphere to be highly dynamic, while the second - standard models, based on the semi-empirical fits to temporally and spatially averaged chromospheric continua and line intensities, - are static (FAL 1 - 3) and assume that the dynamic effects are of minor importance in atmosphere structuring.

The analysis of the dynamic simulations of Carlsson \& Stein reveals that radio emission (brightness temperature contribution functions and brightness spectrum) at millimeter wavelengths is extremely sensitive to the dynamic processes 


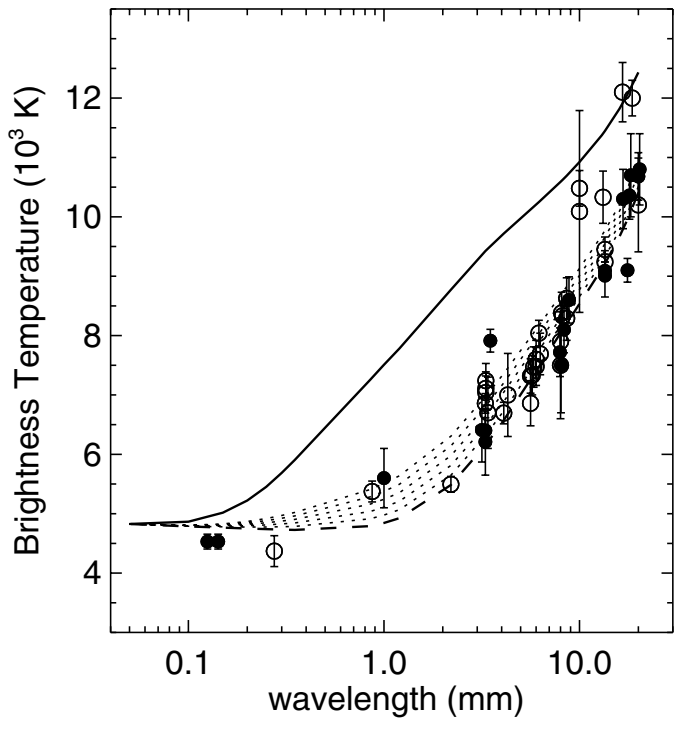

Fig. 9. Comparison of the time-averaged spectrum of the $C S$ model (dashed curve) with observations made at solar cycle minimum (filled circles) and solar maximum (open circles). The spectrum of the $F A L F$ model is represented by the solid curve. Thin dotted curves represent brightness temperature spectra for the $F A L F+C S$ combination, with $F A L F$ filling factor values from 0.05 up to 0.25 in steps of 0.05 .

in the chromosphere, if these are spatially and temporally resolved. In particular, dynamic effects drastically alter the height at which the dominant contributions to emission occur. The spectrum obtained by averaging over the spectra from all timesteps of $C S$ simulations provides a good fit to observed temporally and spatially averaged millimeter data in spite of the absence of the temperature rise at low chromospheric heights in it. At radio wavelengths the source function is linear in the temperature but the electron densities are much higher than in equilibrium models of the same temperature. Hence, the dynamic picture of the solar internetwork chromosphere is consistent with millimeter and submillimeter brightness observations. In contrast, the standard average quiet Sun model, FALC is slightly too bright at millimeter wavelengths. The internetwork model $F A L A$, however, provides a good fit to the data. Thus UV and millimeter data can be simultaneously reproduced only by a combination of model atmospheres and not by a single $F A L$ model.

In spite of the inability of currently available millimeter wavelength data to clearly distinguish between the two very different pictures of the chromosphere, our calculations indicate that such data hold great promise for the future. The main handicap of the current data is their poor spatial resolution, so that most observations average over multiple supergranules and over many patches of coherent shock-wave propagation. Hence, the signature of local chromospheric dynamics is greatly smeared out. Nonetheless local intensity variations in the solar submillimeter (Lindsey \& Kaminski 1984) and millimeter (Simon \& Shimabukuro 1971) continuum are seen in such observations (spatial resolution of several square arc minutes).
Figures 3 and 4 demonstrate that at high spatial and temporal resolution millimeter observations will reveal the signature of shock waves very clearly, if they are present. Observations in this wavelength range with high temporal and spatial resolution, obtained, e.g. interferometrically using BIMA (BerkeleyIllinois-Maryland Millimeter Array), and in future at even higher spatial resolution with the Atacama Large Millimeter Array (ALMA), may thus turn out to be a key to determining the importance of chromospheric dynamics and thus possibly settling the question of the nature of the chromosphere.

Acknowledgements. M. Loukitcheva gratefully acknowledges the hospitality of the staff at the Max-Planck-Institut für Aeronomie. Part of this work was supported by the Russian scientific programs "Astronomy" (grant 1.5.2.2), "Integration" (project AO1072.1/2000) and INTAS (grant 01-543). This work was supported by the Norwegian Research Council's grant 146467/420, "Solar and Heliospheric Plasmas", by NASA grants NAG 59563 and NAG 5 12450, and by NSF grant AST 0205500.

\section{References}

Athay, R. G. 1976, in The Solar Chromosphere and Corona: Quiet Sun (Dordrecht: Reidel), 227

Ayres, T. R., Testerman, L., \& Brault, J. W. 1986, ApJ, 304, 542

Avrett, E. H. 1985, in Chromospheric diagnostics and modelling, ed. B. W. Lites, National Solar Observatory, Sunspot, 67

Carlsson, M. 1992, in ASP Conf. Ser. 26, ed. M. S. Giampapa, \& J. A. Bookbinder, 499

Carlsson, M., \& Stein, R. F. 1992, ApJ, 397, L59

Carlsson, M., \& Stein, R. F. 1995, ApJ, 440, L29

Carlsson, M., \& Stein, R. F. 1997, ApJ, 481, 500

Carlsson, M., \& Stein, R. F. 2002, ApJ, 572, 626

Carlsson, M., Judge, P. G., \& Wilhelm, K. 1997, ApJ, 486, L63

Cram, L. E., \& Damé, L. 1983, ApJ, 272, 355

Fedoseev, L. I., \& Chernyshev, V. I. 1998, Astron. Rep., 42, 105

Fontenla, J. M., Avrett, E. H., \& Loeser, R. 1993, ApJ, 406, 319

Fürst, E. 1980, in Radio Physics of the Sun, ed. M. R. Kundu, \& T. E. Gergely (Dordrecht: Reidel), 25

Hagen, J. 1951, ApJ, 113, 547

Kislyakov, A. G., \& Kuznetsova, N. A. 1977, Solnechnye Dannye, 8, 71

Lindsey, C., \& Kaminski, G. 1984, ApJ, 282, L103

Lites, B. W., Rutten, R. J., \& Kalkofen, W. 1993, ApJ, 414, 345

Maltby, P., Avrett, E. H., Carlsson, M., et al. 1986, ApJ, 306, 284

Nagnibeda, V. G., \& Piotrovich, V. V. 1987, Publications of AO of Leningrad State University, 41, 5

Pelyushenko, S. A. 1982, Izv. Visshikh Uchebn. Zavedenii, Radiofizika, 25, 977

Rutten, R. J., \& Uitenbroek, H. 1991, Sol. Phys., 134, 15

Simon, M., \& Shimabukuro, F. I. 1971, ApJ, 168, 525

Solanki, S. K., Steiner, O., \& Uitenbroek, H. 1991, A\&A, 250, 220

Stallcop, J. R. 1974, ApJ, 187, 179

Vernazza, J. E., Avrett, E. H., \& Loeser, R. 1981, ApJS, 46, 635

Warren, H. P., Mariska, J. T., \& Wilhelm, K. 1998, ApJS, 119, 105

Wilhelm, K., \& Kalkofen, W. 2003, A\&A, 408, 1137

Zheleznyakov, V. V. 1996, Radiation in Astrophysical Plasmas

(Dordrecht: Kluwer Academic Publishers), Chap. 6

Zirin, H., Baumert, B. M., \& Hurford, G. J. 1991, ApJ, 370, 779

Zlotnik, E. Y. 1968, AZh, 45, 310, 585 


\section{Online Material}


Table 1. Observed brightness temperatures with uncertainties, dates of observation, solar cycle phase, beam size of the used instruments, the method of observation and calibration and the references.

\begin{tabular}{|c|c|c|c|c|c|c|c|}
\hline$\lambda(\mathrm{mm})$ & $T[\mathrm{~K}]$ & $\pm[\mathrm{K}]$ & Year & Phase & $\theta\left[{ }^{\prime}\right]$ & Method & Source \\
\hline 0.13 & 4530 & 125 & 1975 & $\mathrm{~m}$ & - & e & Rast, J., Kneubühl, F. K., \& Müller, E. A. 1978, A\&A, 68, 229 \\
\hline 0.14 & 4530 & 125 & 1975 & $\mathrm{~m}$ & - & $\mathrm{e}$ & Rast, J., Kneubühl, F. K., \& Müller, E. A. 1978, A\&A, 68, 229 \\
\hline 0.28 & 4370 & 260 & 1968 & M & - & $\mathrm{h}$ & $\begin{array}{l}\text { Eddy, J. A., Lena, P. J., \& McQueen, R. M. 1969, Sol. Phys., } \\
10,330\end{array}$ \\
\hline 0.34 & 4540 & 360 & 1972 & $\mathrm{i}$ & 2.00 & $\mathrm{~b}$ & Gesari, P. T., Joyce, R. R., \& Simon, M. 1973, A\&A, 26, 409 \\
\hline 0.45 & 4800 & 380 & 1972 & $\mathrm{i}$ & 2.00 & $\mathrm{~b}$ & Gesari, P. T., Joyce, R. R., \& Simon, M. 1973, A\&A, 26, 409 \\
\hline 0.74 & 5200 & 1000 & 1966 & $\mathrm{i}$ & 10.00 & g & $\begin{array}{l}\text { Fedoseev, L. I., Lubyako, L. V., \& Kuklik, L. M. 1967, AZh, } \\
44,1191\end{array}$ \\
\hline 0.85 & 6400 & 700 & 1991 & $\mathrm{i}$ & 0.30 & $\mathrm{~d}$ & Ewell, M., Zirin, J., \& Jensen, J. 1993, ApJ, 403, 426 \\
\hline 0.87 & 5960 & 800 & 1966 & $\mathrm{i}$ & 12.50 & $\mathrm{f}$ & $\begin{array}{l}\text { Fedoseev, L. I., Lubyako, L. V., \& Kuklik, L. M. 1967, AZh, } \\
44,1191\end{array}$ \\
\hline 0.87 & 5374 & 176 & 1979 & M & 4.00 & $\mathrm{c}$ & $\begin{array}{l}\text { Fedoseev, L. I., \& Chernyshev, V. I. 1998, Astronomy Rep., 42, } \\
105\end{array}$ \\
\hline 1.00 & 5600 & 400 & 1972 & $\mathrm{i}$ & 7.00 & $\mathrm{~b}$ & Gesari, P. T., Joyce, R. R., \& Simon, M. 1973, A\&A, 26, 409 \\
\hline 1.00 & 5600 & 500 & 1964 & $\mathrm{~m}$ & 3.90 & a & Low, F. G., \& Davidson, A. W. 1965, ApJ, 142, 1278 \\
\hline 1.06 & 6620 & 600 & 1966 & $\mathrm{i}$ & 14.00 & $\mathrm{f}$ & $\begin{array}{l}\text { Fedoseev, L. I., Lubyako, L. V., \& Kuklik, L. M. 1967, AZh, } \\
44,1191\end{array}$ \\
\hline 1.20 & 6100 & 400 & 1961 & $\mathrm{i}$ & 3.00 & a & $\begin{array}{l}\text { Bastin, J. A., Gear, A. E., Jones, G. O., et al. 1964, Proc. Roy. } \\
\text { Soc. London, A, 278, } 543\end{array}$ \\
\hline 1.26 & 6930 & 500 & 1966 & $\mathrm{i}$ & 10.00 & $\mathrm{f}$ & $\begin{array}{l}\text { Fedoseev, L. I., Lubyako, L. V., \& Kuklik, L. M. 1967, AZh, } \\
44,1191\end{array}$ \\
\hline 1.30 & 6011 & 240 & 1973 & $\mathrm{i}$ & 0.70 & $\mathrm{c}$ & Wrixon, G. T., \& Schneider, M. V. 1974, Nature, 250, 314 \\
\hline 1.30 & 6430 & 400 & 1961 & $\mathrm{i}$ & 3.00 & a & $\begin{array}{l}\text { Bastin, J. A., Gear, A. E., Jones, G. O., et al. 1964, Proc. Roy. } \\
\text { Soc. London, A, 278, } 543\end{array}$ \\
\hline 1.30 & 6000 & 500 & 1961 & $\mathrm{i}$ & 3.00 & a & $\begin{array}{l}\text { Bastin, J. A., Gear, A. E., Jones, G. O., et al. 1964, Proc. Roy. } \\
\text { Soc. London, A, 278, } 543\end{array}$ \\
\hline 1.80 & 6030 & 700 & 1962 & $\mathrm{i}$ & 9.00 & e & $\begin{array}{l}\text { Naumov, A. I. 1963, Izv. Visshikh Uchebn. Zavedenii, } \\
\text { Radiofizika, } 6,848\end{array}$ \\
\hline 2.00 & 5670 & 230 & 1961 & $\mathrm{i}$ & 7.00 & $\mathrm{f}$ & Wort, D. 1962 , Nature, 195,1288 \\
\hline 2.15 & 6140 & 500 & 1960 & $\mathrm{i}$ & 6.00 & $\mathrm{f}$ & Tolbert, C. W., \& Straiton, A. W. 1961, ApJ, 134, 91 \\
\hline 2.20 & 5495 & 133 & 1979 & M & 3.50 & $\mathrm{c}$ & $\begin{array}{l}\text { Fedoseev, L. I., \& Chernyshev, V. I. 1998, Astronomy Rep., 42, } \\
105\end{array}$ \\
\hline 2.20 & 7410 & 400 & 1961 & $\mathrm{i}$ & 5.00 & a & $\begin{array}{l}\text { Bastin, J. A., Gear, A. E., Jones, G. O., et al. 1964, Proc. Roy. } \\
\text { Soc. London, A, 278, } 543\end{array}$ \\
\hline 2.40 & 7080 & 400 & 1961 & $\mathrm{i}$ & 5.00 & a & $\begin{array}{l}\text { Bastin, J. A., Gear, A. E., Jones, G. O., et al. 1964, Proc. Roy. } \\
\text { Soc. London, A, 278, } 543\end{array}$ \\
\hline 2.73 & 6220 & 715 & 1960 & $\mathrm{i}$ & 8.00 & $\mathrm{f}$ & Tolbert, C. W., \& Straiton, A. W. 1961, ApJ, 134, 91 \\
\hline 2.80 & 7410 & 500 & 1961 & $\mathrm{i}$ & 6.00 & a & $\begin{array}{l}\text { Bastin, J. A., Gear, A. E., Jones, G. O., et al. 1964, Proc. Roy. } \\
\text { Soc. London, A, 278, } 543\end{array}$ \\
\hline 3.00 & 6640 & 950 & 1960 & $\mathrm{i}$ & 9.00 & $\mathrm{f}$ & Tolbert, C. W., \& Straiton, A. W. 1961, ApJ, 134, 91 \\
\hline 3.10 & 6815 & 310 & 1971 & $\mathrm{i}$ & 2.52 & a & Ulich, B. L., Cogdell, J. R., \& Davis, J. H. 1973, Icarus, 19, 59 \\
\hline 3.17 & 6410 & 540 & 1975 & $\mathrm{~m}$ & 7.00 & $\mathrm{c}$ & Kuseski, R. A., \& Swanson, P. N. 1976, Sol. Phys., 48, 41 \\
\hline 3.30 & 6402 & 215 & 1964 & $\mathrm{~m}$ & 3.00 & $\mathrm{~g}$ & Simon, M. 1965, ApJ, 141, 1513 \\
\hline 3.30 & 6210 & 560 & 1975 & $\mathrm{~m}$ & 7.00 & $\mathrm{c}$ & Kuseski, R. A., \& Swanson, P. N. 1976, Sol. Phys., 48, 41 \\
\hline 3.30 & 6858 & 165 & 1979 & M & 5.00 & $\mathrm{c}$ & $\begin{array}{l}\text { Fedoseev, L. I., \& Chernyshev, V. I. 1998, Astronomy Rep., 42, } \\
105\end{array}$ \\
\hline 3.32 & 7040 & 280 & 1969 & M & 2.80 & $\mathrm{c}$ & Reber, E. E. 1971, Sol. Phys., 16, 75 \\
\hline 3.32 & 7240 & 290 & 1969 & M & 2.80 & $\mathrm{c}$ & Reber, E. E. 1971, Sol. Phys., 16, 75 \\
\hline 3.32 & 7110 & 280 & 1969 & M & 2.80 & $\mathrm{c}$ & Reber, E. E. 1971, Sol. Phys., 16, 75 \\
\hline 3.40 & 6900 & 400 & 1991 & $\mathrm{i}$ & 2.50 & $\mathrm{c}$ & $\begin{array}{l}\text { Nagnibeda, V. G., \& Piotrovitch, V. V. 1994, Sol. Phys., 152, } \\
175\end{array}$ \\
\hline 3.50 & 7914 & 192 & 1977 & $\mathrm{~m}$ & 3.00 & $\mathrm{~g}$ & $\begin{array}{l}\text { Ulich, B. L., Davis, J. H., Phodes, P. J., et al. 1980, IEEE } \\
\text { Transactions on antennas and propagation, AP- } 28,3,367\end{array}$ \\
\hline 4.10 & 6695 & 182 & 1979 & M & 9.00 & $\mathrm{c}$ & $\begin{array}{l}\text { Fedoseev, L. I., \& Chernyshev, V. I. 1998, Astronomy Rep., 42, } \\
105\end{array}$ \\
\hline
\end{tabular}


Table 1. continued.

\begin{tabular}{|c|c|c|c|c|c|c|c|}
\hline$\lambda(\mathrm{mm})$ & $T[\mathrm{~K}]$ & $\pm[\mathrm{K}]$ & Year & Phase & $\theta\left[{ }^{\prime}\right]$ & Method & Source \\
\hline 4.30 & 7000 & 700 & 1957 & M & 6.70 & g & Coates, R. J. 1958, Proc. of IRE, 46, 122 \\
\hline 5.62 & 7320 & 290 & 1969 & M & 4.20 & $\mathrm{c}$ & Reber, E. E. 1971, Sol. Phys., 16, 75 \\
\hline 5.62 & 6860 & 380 & 1969 & M & 4.20 & $\mathrm{c}$ & Reber, E. E. 1971, Sol. Phys., 16, 75 \\
\hline 5.73 & 7330 & 330 & 1969 & M & 4.30 & $\mathrm{c}$ & Reber, E. E. 1971, Sol. Phys., 16, 75 \\
\hline 5.83 & 7470 & 340 & 1969 & M & 4.40 & $\mathrm{c}$ & Reber, E. E. 1971, Sol. Phys., 16, 75 \\
\hline 6.00 & 7600 & 320 & 1969 & M & 4.50 & $\mathrm{c}$ & Reber, E. E. 1971, Sol. Phys., 16, 75 \\
\hline 6.00 & 7480 & 320 & 1969 & M & 4.50 & $\mathrm{c}$ & Reber, E. E. 1971, Sol. Phys., 16, 75 \\
\hline 6.00 & 4500 & 400 & 1956 & $\mathrm{i}$ & - & $\mathrm{f}$ & $\begin{array}{l}\text { Whitehurst, R. N., Copeland, J., \& Mitchell, F. H. 1957, J. Appl. } \\
\text { Phys., 28, } 295\end{array}$ \\
\hline 6.20 & 8041 & 216 & 1979 & M & 10.50 & $\mathrm{c}$ & $\begin{array}{l}\text { Fedoseev, L. I., \& Chernyshev, V. I. 1998, Astronomy Rep., 42, } \\
105\end{array}$ \\
\hline 6.30 & 7690 & 350 & 1979 & M & 11.00 & $\mathrm{c}$ & Pelyushenko, S. A., \& Chernyshev, V. I. 1983, AZh, 60, 584 \\
\hline 7.95 & 7720 & 410 & 1975 & $\mathrm{~m}$ & 14.00 & $\mathrm{c}$ & Kuseski, R. A., \& Swanson, P. N. 1976, Sol. Phys., 48, 41 \\
\hline 8.00 & 7500 & 900 & 1957 & M & 2.00 & $\mathrm{a}$ & Salomonovich, A. E. 1962, AZh, 39, 260 \\
\hline 8.03 & 7590 & 350 & 1974 & $\mathrm{i}$ & 14.00 & $\mathrm{c}$ & Swanson, P. N., \& Kuseski, R. A. 1975, Nature, 253, 513 \\
\hline 8.04 & 7500 & 800 & 1964 & $\mathrm{~m}$ & 1.60 & $\mathrm{a}$ & Efanov, V. A., \& Moiseev, I. G. 1965, Izv. CRAO, 34, 53 \\
\hline 8.10 & 8380 & 350 & 1969 & M & 1.70 & $\mathrm{c}$ & Efanov, V. A., \& Moiseev, I. G. 1971, Izv. CRAO, 43, 21 \\
\hline 8.20 & 8333 & 248 & 1979 & M & 14.00 & $\mathrm{c}$ & $\begin{array}{l}\text { Fedoseev, L. I., \& Chernyshev, V. I. 1998, Astronomy Rep., 42, } \\
105\end{array}$ \\
\hline 8.33 & 8100 & 390 & 1975 & $\mathrm{~m}$ & 14.00 & $\mathrm{c}$ & Kuseski, R. A., \& Swanson, P. N. 1976, Sol. Phys., 48, 41 \\
\hline 8.33 & 8000 & 350 & 1974 & $\mathrm{i}$ & 14.00 & $\mathrm{c}$ & Swanson, P. N., \& Kuseski, R. A. 1975, Nature, 253, 513 \\
\hline 8.50 & 6740 & 674 & 1948 & $\mathrm{i}$ & 12.00 & $\mathrm{f}$ & Hagen, J. 1951, ApJ, 113, 547 \\
\hline 8.60 & 7250 & 256 & 1971 & $\mathrm{i}$ & 6.60 & $\mathrm{~g}$ & Ulich, B. L., Cogdell, J. R., \& Davis, J. H. 1973, Icarus, 19, 59 \\
\hline 8.60 & 8290 & 370 & 1979 & M & 15.00 & $\mathrm{c}$ & Pelyushenko, S. A., \& Chernyshev, V. I. 1983, AZh, 60, 584 \\
\hline 8.60 & 8627 & 347 & 1979 & M & 15.00 & $\mathrm{c}$ & $\begin{array}{l}\text { Fedoseev, L. I., \& Chernyshev, V. I. 1998, Astronomy Rep., 42, } \\
105\end{array}$ \\
\hline 10.0 & 10090 & 1700 & 1968 & M & - & $\mathrm{h}$ & $\begin{array}{l}\text { El Raye, M., Silver, S., \& Welch, W. J. 1969, Solar Emission, } \\
\text { Space Science Lab. Univ. Calf., Series 10, Is. } 55\end{array}$ \\
\hline 11.80 & 10330 & 300 & 1966 & $\mathrm{i}$ & 6.00 & $\mathrm{a}$ & $\begin{array}{l}\text { Staelin, D. H., Gant, N. E., Law, S. E., et al. 1968, Sol. Phys., 3, } \\
26\end{array}$ \\
\hline 11.80 & 9350 & 980 & 1962 & $\mathrm{i}$ & 6.00 & $\mathrm{a}$ & Staelin, D. H., Barret, A. H., \& Kusse, B. R. 1964, AJ, 69, 69 \\
\hline 12.80 & 11260 & 500 & 1966 & $\mathrm{i}$ & 6.40 & a & $\begin{array}{l}\text { Staelin, D. H., Gant, N. E., Law, S. E., et al. 1968, Sol. Phys., 3, } \\
26\end{array}$ \\
\hline 13.00 & 10400 & 1230 & 1992 & $\mathrm{i}$ & 2.00 & a & Bastian, T. S., Dulk, G. A., \& Leblanc, Y. 1996, ApJ, 473, 539B \\
\hline 13.20 & 10330 & 440 & 1969 & M & 2.50 & $\mathrm{c}$ & Efanov, V. A., \& Moiseev, I. G. 1971, Izv. CRAO, 43, 21 \\
\hline 13.50 & 9240 & 190 & 1978 & M & 2.55 & $\mathrm{a}$ & $\begin{array}{l}\text { Efanov, V. A., Moiseev, I. G., Nesterov, N. S., \& Stewart, R. T. } \\
\text { 1980, in Radio Physics of the Sun, ed. M. R. Kundu, \& T. E. } \\
\text { Gergely, Reidel, Dordrecht, } 141\end{array}$ \\
\hline 13.50 & 9450 & 100 & 1973 & $\mathrm{i}$ & 2.55 & $\mathrm{a}$ & $\begin{array}{l}\text { Efanov, V. A., Moiseev, I. G., Nesterov, N. S., \& Stewart, R. } \\
\text { T. 1980, in Radio Physics of the Sun, ed. M. R. Kundu, T. E. } \\
\text { Gergely, Reidel, Dordrecht, } 141\end{array}$ \\
\hline 13.50 & 9450 & 210 & 1979 & M & 2.55 & $\mathrm{a}$ & $\begin{array}{l}\text { Efanov, V. A., Moiseev, I. G., Nesterov, N. S., \& Stewart, R. T. } \\
\text { 1980, in Radio Physics of the Sun, ed. M. R. Kundu, \& T. E. } \\
\text { Gergely, Reidel, Dordrecht, } 141\end{array}$ \\
\hline 13.50 & 11560 & 500 & 1966 & $\mathrm{i}$ & 6.80 & $\mathrm{a}$ & $\begin{array}{l}\text { Staelin, D. H., Gant, N. E., Law, S. E., et al. 1968, Sol. Phys., 3, } \\
26\end{array}$ \\
\hline 13.50 & 9090 & 150 & 1976 & $\mathrm{~m}$ & 2.55 & $\mathrm{a}$ & $\begin{array}{l}\text { Efanov, V. A., Moiseev, I. G., Nesterov, N. S., \& Stewart, R. T. } \\
\text { 1980, in Radio Physics of the Sun, ed. M. R. Kundu, \& T. E. } \\
\text { Gergely, Reidel, Dordrecht, } 141\end{array}$ \\
\hline 13.50 & 9010 & 360 & 1977 & $\mathrm{~m}$ & 2.55 & $\mathrm{a}$ & $\begin{array}{l}\text { Efanov, V. A., Moiseev, I. G., Nesterov, N. S., \& Stewart, R. T. } \\
\text { 1980, in Radio Physics of the Sun, ed. M. R. Kundu, \& T. E. } \\
\text { Gergely, Reidel, Dordrecht, } 141\end{array}$ \\
\hline
\end{tabular}


Table 1. continued.

\begin{tabular}{|c|c|c|c|c|c|c|c|}
\hline$\lambda(\mathrm{mm})$ & $T[\mathrm{~K}]$ & $\pm[\mathrm{K}]$ & Year & Phase & $\theta\left[{ }^{\prime}\right]$ & Method & Source \\
\hline 14.30 & 11350 & 400 & 1966 & $\mathrm{i}$ & 7.10 & a & $\begin{array}{l}\text { Staelin, D. H., Gant, N. E., Law, S. E., et al. 1968, Sol. Phys., } 3 \text {, } \\
26\end{array}$ \\
\hline 15.80 & 11330 & 400 & 1966 & $\mathrm{i}$ & 7.90 & a & $\begin{array}{l}\text { Staelin, D. H., Gant, N. E., Law, S. E., et al. 1968, Sol. Phys., } 3 \text {, } \\
26\end{array}$ \\
\hline 16.60 & 12100 & 500 & 1969 & M & 3.00 & $\mathrm{c}$ & Efanov, V. A., \& Moiseev, I. G. 1971, Izv. CRAO, 43, 21 \\
\hline 16.70 & 10300 & 500 & 1986 & $\mathrm{~m}$ & 2.60 & a & Zirin, H., Baumert, B. M., \& Hurford, G. J. 1991, ApJ, 370, 779 \\
\hline 18.30 & 10700 & 700 & 1986 & $\mathrm{~m}$ & 2.84 & a & Zirin, H., Baumert, B. M., \& Hurford, G. J. 1991, ApJ, 370, 779 \\
\hline 19.00 & 9350 & 320 & 1974 & $\mathrm{i}$ & 2.00 & $\mathrm{c}$ & Bachurin, A. F., \& Eryushev, N. N. 1976, Izv. CRAO, 54, 241 \\
\hline 19.00 & 9980 & 350 & 1972 & $\mathrm{i}$ & 2.00 & $\mathrm{c}$ & $\begin{array}{l}\text { Bachurin, A. F., Eryushev, N. N., \& Tsvetkov, L. I. 1974, Izv } \\
\text { CRAO, 50, } 180\end{array}$ \\
\hline 19.00 & 10000 & 320 & 1973 & $\mathrm{i}$ & 2.00 & $\mathrm{c}$ & Bachurin, A. F., \& Eryushev, N. N. 1976, Izv. CRAO, 54, 241 \\
\hline 19.00 & 10130 & 320 & 1973 & $\mathrm{i}$ & 2.00 & $\mathrm{c}$ & Bachurin, A. F., \& Eryushev, N. N. 1976, Izv. CRAO, 54, 241 \\
\hline 20.00 & 12890 & 1415 & 1992 & $\mathrm{i}$ & 3.00 & a & Bastian, T. S., Dulk, G. A., \& Leblanc, Y. 1996, ApJ, 473, 539B \\
\hline 20.00 & 10200 & 790 & 1968 & M & 2.00 & $\mathrm{c}$ & Buhl, D., \& Tlamicha, A. 1970, A\&A, 5, 102 \\
\hline 20.30 & 10800 & 600 & 1986 & $\mathrm{~m}$ & 3.10 & a & Zirin, H., Baumert, B. M., \& Hurford, G. J. 1991, ApJ, 370, 779 \\
\hline
\end{tabular}

